\title{
Frequency of Bonded Bracket Failure in Patients, Undergoing Fixed Orthodontic Treatment
}

$\begin{array}{ll}\text { Hasnain Sakrani }^{1} & \text { BDS, FCPS } \\ \text { Sabeen Masood }^{2} & \text { BDS } \\ \text { Fiza Bibi Alavi }^{3} & \text { BDS } \\ \text { Mustafa Dahar }^{4} & \text { BDS } \\ \text { Mahnoor Khawaja M. Saleem }^{5} & \text { BDS } \\ \text { Abhishek Lal }^{6} & \text { BDS }\end{array}$

\author{
BDS, FCPS \\ BDS \\ BDS \\ BDS \\ BDS
}

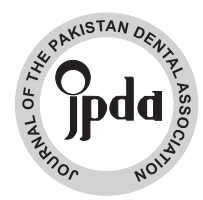

OBJECTIVE: The objective of our study is to determine frequency of bracket bond failure in relation to age, gender, most commonly involved tooth and quadrant.

METHODOLOGY: In this descriptive cross-sectional study, a total of 100 patients with ages ranging from 10 to 30 years needing corrective orthodontic treatment were selected for the study. This survey was conducted at Altamash Institute of Dental Medicine, Karachi. The survey was initiated on 30th November 2019 and was concluded on 30th May 2020. A questionnaire was used to evaluate the frequency of bonded bracket failure in patients undergoing orthodontic treatment. The bonded metallic brackets were light cured for 40 seconds, and initial alignment arch wires were inserted. Bonded bracket failure was recorded over a period of six months.

RESULTS: The initial debonding results of the present study demonstrated a high number of debonded brackets in mandibular dentition as compared to the maxillary dentition. Whereas, when the teeth were debonded for the second time, a greater number of maxillary teeth were found to be affected by the debonding of the brackets. Both genders demonstrated a greater rate of bracket debonding in the second premolar area and lowest in the molar area. In our study, age and gender had no significant relationship with bracket bond failure and teeth involved in debonding.

CONCLUSIONS: The frequency of bonded bracket detachment during orthodontic treatment is very common. Our study concluded that females were more affected by the bracket bond failure as compared to the males along with the second premolar being the most commonly associated tooth during an active orthodontic treatment.

KEYWORDS: Bonded brackets, Bracket failure, Frequency, Orthodontic treatment, Orthodontics

HOW TO CITE: Sakrani H, Masood S, Alavi FB, Dahar M, Saleem MKM, Lal A. Frequency of bonded bracket failure in patients, undergoing fixed orthodontic treatment . J Pak Dent Assoc 2021;30(3):189-193.

DOI: https://doi.org/10.25301/JPDA.303.189

Received: 24 October 2020, Accepted: 30 April 2021

\section{INTRODUCTION}

A well-aligned ideal occlusion is not always present in every individual, with many facing malocclusion problems whether being confined to a single tooth or being generalized throughout the dentition. ${ }^{1}$ Orthodontic treatment in recent years has been emerging as one of the

1. Professor, Depertment of Orthodontics, Altamash Institute of Dental Medicine, Karachi.

2. FCPS Resident, Department of Operative Dentistry, Altamash Institute of Dental Medicine, Karachi.

3. Graduate, Altamash Institute of Dental Medicine, Karachi.

4. Graduate, Altamash Institute of Dental Medicine, Karachi.

5. Graduate, Final Professional, Bachelor of Dental Surgery, Altamash Institute of Dental Medicine, Karachi.

6. Graduate, Final Professional, Bachelor of Dental Surgery, Altamash Institute of Dental Medicine, Karachi.

Corresponding author: "Abhishek Lal” < abhishekdarshan@yahoo.com > most sought out dental treatment not only opted by young individuals but older individuals as well. One of the prime reasons for the people having high expectations is the advertisements being shown on television screens with perfectly well-aligned and brighter looking teeth. Apart from physical attractiveness, people having other kinds of expectations from their practitioners include better masticatory functions eventually leading to some improvement in speech. ${ }^{2}$ When there is a deviation from the normally developing dentofacial structures, there is a high chance of having a negative impact on the individual's life which psychologically affects his quality of life, selfimage, and self-esteem. ${ }^{3}$ These all factors then necessitate an individual to opt for orthodontic treatment to solve these problems. 
Three-dimensional control of teeth during orthodontic treatment plays an important role in achieving optimum treatment results. ${ }^{4}$ Tooth movement can be achieved either firstly with the use of fixed appliances and is a result of the interaction between the bracket on the tooth surface and the arch wires, which are tied into each bracket, and secondly, with removable appliances. ${ }^{5}$ Fixed appliances are a better option for the patient, especially in children as compliance is a major issue to make the treatment effective. Adults, on the other hand, are more mature to understand the part to be played on their end to achieve the desired results. A crucial part of any treatment for the patients, especially orthodontic treatment, motivation by the patient to get the desired results at the end of the treatment is of paramount importance. $^{6}$

Premature debonding of brackets during fixed appliance treatment can be an inconvenience to the patient and the clinician and can result from poor patient compliance, poor clinical bonding technique, or use of unsatisfactory bonding materials. ${ }^{7}$ Usually, bonding is done with the application of $37 \%$ phosphoric acid on tooth enamel for 15 seconds followed by the application of unfilled composite resin and placement of the bracket on the tooth surface having filled composite resin at its base. Composite resin is cured with the help of either light-cure or chemical cure initiation. ${ }^{8}$ The success of bonding is dependent on the bonding technique used, the concentration of etchant gel and application time of etchant, bracket base structure and, operator expertise. Patient factors which also include eating habits play an important role in preventing bracket bond failure. ${ }^{9}$ Investigators have previously studied the prevalence of bracket bond failure to gender and the site of bond failure. ${ }^{10}$ Researchers have also studied the bond strength of different bonding materials on brackets and the type of bonding technique used which may be either direct or indirect. ${ }^{11}$ However, in our research, we investigated the frequency of bracket bond failure in relation to age, gender, most commonly involved tooth and quadrant.

To evaluate the occurrence of debonding in the patients, in this study, we evaluated the relationship of age and gender with debonding of brackets, specific teeth on which debonding occurred, arch where debonding most commonly occurred, and the duration between the episodes of debonding of brackets. Debonding occurring for the second time was also evaluated with similar variables as used for the initial debonding of the brackets.

\section{METHODOLOGY}

This is a descriptive cross-sectional study that is based in one of the leading institutions and was conducted from
30 November 2019 to 30 May 2020 to determine the Frequency of bonded bracket failure in patients, undergoing fixed orthodontic treatment at the Department of orthodontics, Altamash Institute of Dental Medicine. The Ethics committee of the institute supervised the study protocol.(AIDM/EC/10/2020/21) Sample size of 100 was calculated by using the WHO online sample size calculator. The data was collected using a self-administered structured questionnaire through convenience sampling and was analyzed by using SPSS version 20. Spearman's correlation test was used to calculate the p-value $<0.05$ which was considered significant. Following the signing of informed consent, a total of 100 cases were included in the study, out of which, 47 (47\%) were males while $53(53 \%)$ were females. Patients with ages ranging from 10 to 30 years needing corrective orthodontic treatment were selected. The operators who participated in this study were 6 in total. Amongst which, 3 were asked to collect the sample size by filling the questionnaires and the remaining did the calculations of the sample size and also contributed in the written part of the research. The procedure of bonding was performed by selected house officers, demonstrators, and resident trainees of the department of orthodontics.

The questionnaire that was given comprised of two main parts. The first part consisted of questions about the candidate's demographic status including age group, gender, opd number, and date of bonding. The second part consisted of questions related to the frequency of debonding, bonding material, arch where the debonding occured, teeth, quadrant, bracket material, and debonding date. A record sheet of every patient was maintained. The data was recorded from the date of initial bonding to the date where the teeth were debonded for the first time and then for the second time irrespective of any fixed duration of time.

\section{Sample selection \\ Inclusion criteria:}

- Both males and females

- Age limit (10-30 years)

- Same brackets set (metallic)

- Same bonding material (light cure composite)

\section{Exclusion criteria:}

- Bonding material other than light cure composite (self-cure composite)

- Brackets set other than metallic (ceramic, composite)

\section{RESULTS}

In this cross-sectional study, a total of 100 patients were included. The mean average age of the patients was 
Sakrani H/ Masood S/ Alavi FB/

Dahar M/ Saleem MKM/ Lal A

$18.78 \pm 5.55$ with the age brackets of $10-15,16-20,21-25$, and 26-30 years old as shown in Table 1. Descriptive statistics along with spearman's correlation test was used to analyze a significant relationship between age, gender, debonded teeth and location of the debonded teeth.

Table 1: Demographic characteristics of the participants $(n=100)$

\begin{tabular}{llll}
\hline & Variables & $\mathbf{n}$ & $\%$ \\
\hline Age & $\mathbf{1 0 - 1 5}$ & 39 & $\mathbf{3 9 . 0}$ \\
& years & 28 & $\mathbf{2 8 . 0}$ \\
& $16-20$ & 17 & 17.0 \\
years & 16 & 16.0 \\
& $21-25$ & & \\
years & & \\
& $26-30$ & & \\
& years & & 47.0 \\
\hline \multirow{6}{*}{ Gender } & Male & 47 & $\mathbf{5 3 . 0}$ \\
& Female & 53 &
\end{tabular}

Regarding the location of the brackets which were initially debonded, 51 (51.5\%) debonded brackets were witnessed in cases involving the mandibular arch only, 39 (39.4\%) involving only the maxillary arch, and 9 (9.1\%) were present in cases involving both maxilla and mandible. In these initial debonded brackets, out of the total sample size which is 100 on which debonding of brackets was encountered included 26 (17.33\%) Central incisor, 26 (17.33\%) Lateral incisor, 29 (19.33\%) Canine, 25 (16.67\%) First Premolar, 38 (25.33\%) Second Premolar, and 6 (4\%) First Molar as shown in table 2.

Table 2: Frequencies of the teeth involved in initial debonding

\begin{tabular}{|l|l|l|}
\hline Location & Teeth & Frequency (Percentage) \\
\hline \multirow{5}{*}{ Maxilla } & Central Incisor & $12(15.2 \%)$ \\
\cline { 2 - 3 } & Lateral Incisor & $13(16.4 \%)$ \\
\cline { 2 - 3 } & Canine & $16(20.2 \%)$ \\
\cline { 2 - 3 } & First Premolar & $16(20.2 \%)$ \\
\cline { 2 - 3 } & Second Premolar & $21(26.6 \%)$ \\
\cline { 2 - 3 } & First Molar & $1(1.26 \%)$ \\
\hline \multirow{5}{*}{ Mandible } & Central Incisor & $14(19.7 \%)$ \\
\cline { 2 - 3 } & Lateral Incisor & $13(16.4 \%)$ \\
\cline { 2 - 3 } & Canine & $13(16.4 \%)$ \\
\cline { 2 - 3 } & First Premolar & $\mathbf{9 ( 1 2 . 7 \% )}$ \\
\cline { 2 - 3 } & Second Premolar & $17(21.5 \%)$ \\
\cline { 2 - 3 } & First Molar & $5(6.3 \%)$ \\
\hline
\end{tabular}

The locations in which debonding occurred for the second time included $27(47.4 \%)$ cases involving the mandibular arch only, 29 (50.9\%) involving the maxillary arch only, and 1 (1.8\%) involving both maxilla and mandible. Of the 61 teeth in which debonding of brackets occurred again, the teeth distribution included $5(8.2 \%)$ central incisors, 5 (8.2\%) lateral incisors, 9 (14.8\%) canines, 12
Frequency of bonded bracket failure in patients, undergoing fixed orthodontic treatment

(19.7\%) First Premolars, 28 (45.9\%) Second Premolars, and $2(3.3 \%)$ First molars as shown in table 3.

Table 3: Frequencies of the teeth on which debonding occurred for the second time

\begin{tabular}{|l|l|l|}
\hline Location & Teeth & Frequency (Percentage) \\
\hline \multirow{5}{*}{ Maxilla } & Central Incisor & $\mathbf{4}(12.5 \%)$ \\
\cline { 2 - 3 } & Lateral Incisor & $\mathbf{0}(0 \%)$ \\
\cline { 2 - 3 } & Canine & $\mathbf{5 ( 1 5 . 6 \% )}$ \\
\cline { 2 - 3 } & First Premolar & $\mathbf{7 ( 2 1 . 9 \% )}$ \\
\cline { 2 - 3 } & Second Premolar & $\mathbf{1 6}(50.0 \%)$ \\
\cline { 2 - 3 } & First Molar & $\mathbf{0}(0 \%)$ \\
\hline \multirow{5}{*}{ Mandible } & Central Incisor & $\mathbf{1 ( 3 . 3 4 \% )}$ \\
\cline { 2 - 3 } & Lateral Incisor & $\mathbf{5 ( 1 7 . 2 \% )}$ \\
\cline { 2 - 3 } & Canine & $\mathbf{4 ( 1 3 . 8 \% )}$ \\
\cline { 2 - 3 } & First Premolar & $\mathbf{5 ( 1 7 . 2 \% )}$ \\
\cline { 2 - 3 } & Second Premolar & $\mathbf{1 2}(41.4 \%)$ \\
\cline { 2 - 3 } & First Molar & $\mathbf{2 ( 6 . 9 \% )}$ \\
\hline
\end{tabular}

Regarding the initial debonding, the brackets were debonded in males 47 times with the second premolar being the most common tooth. In females, initial debonding occurred 53 times, of which the second premolar was found to be the most common tooth. About debonding which occurred for the second time, the total cases witnessed were 61 with the second premolar being the most commonly encountered tooth.

Spearman's correlation test was used for the analysis of significant relation of age and gender with bracket bond failure and teeth involved. In our study, debonding location had a significant relationship with debonding teeth ( $\mathrm{p}$-value $=0.004)$. Whereas, age and gender had no significant relationship with bracket bond failure and the teeth involved as shown in Table 4.

Table 4: Spearman's correlation

\begin{tabular}{|c|c|c|c|c|c|c|c|c|}
\hline \multicolumn{9}{|c|}{ Correlations } \\
\hline & & & Age & Gender & $\begin{array}{c}\begin{array}{c}\text { Debonding } \\
\text { Location }\end{array} \\
\text {. }\end{array}$ & $\begin{array}{c}\text { Debonding } \\
\text { Teeth }\end{array}$ & $\begin{array}{c}\text { Again, } \\
\text { Debonding } \\
\text { Location }\end{array}$ & $\begin{array}{c}\text { Again, } \\
\text { Debonding } \\
\text { Teeth }\end{array}$ \\
\hline \multirow[t]{12}{*}{$\begin{array}{l}\text { Spearman's } \\
\text { rho }\end{array}$} & \multirow[t]{2}{*}{ Age } & $\begin{array}{l}\text { Correlation } \\
\text { Coefficient }\end{array}$ & 1.000 & .013 & -.087 & -.010 & -.008 & -.028 \\
\hline & & Sig. (2-tailed) & & .902 & .394 & .924 & .951 & .785 \\
\hline & \multirow[t]{2}{*}{ Gender } & $\begin{array}{l}\text { Correlation } \\
\text { Coefficient }\end{array}$ & .013 & 1.000 & .014 & .094 & -.034 & .101 \\
\hline & & Sig. (2-tailed) & .902 & & .889 & .362 & .801 & .315 \\
\hline & \multirow[t]{2}{*}{$\begin{array}{l}\text { Debonding } \\
\text { Location }\end{array}$} & $\begin{array}{l}\text { Correlation } \\
\text { Coefficient }\end{array}$ & \begin{tabular}{ll|l|}
-087 \\
\end{tabular} & .014 & 1.000 & $-.292^{* *}$ & $.784^{*+}$ & -133 \\
\hline & & Sig. (2-tailed) & .394 & .889 & & .004 & .000 & .189 \\
\hline & \multirow[t]{2}{*}{$\begin{array}{l}\text { Debonding } \\
\text { Teeth }\end{array}$} & $\begin{array}{l}\text { Correlation } \\
\text { Coefficient }\end{array}$ & -.010 & .094 & $-.292^{* *}$ & 1.000 & -.120 & .022 \\
\hline & & Sig. (2-tailed) & .924 & .362 & .004 & & .377 & .834 \\
\hline & \multirow{2}{*}{\begin{tabular}{l|} 
Again, \\
Debonding \\
Location \\
\end{tabular}} & $\begin{array}{l}\text { Correlation } \\
\text { Coefficient }\end{array}$ & \begin{tabular}{ll|l|}
-008 \\
\end{tabular} & $\begin{array}{ll}.034 \\
\end{array}$ & $.784^{* *}$ & -.120 & 1.000 & -.031 \\
\hline & & Sig. (2-tailed) & .951 & .801 & .000 & .377 & & .822 \\
\hline & \multirow{2}{*}{$\begin{array}{l}\text { Again, } \\
\text { Debonding } \\
\text { Teeth }\end{array}$} & $\begin{array}{l}\text { Correlation } \\
\text { Coefficient }\end{array}$ & $\begin{array}{l}-.028 \\
\end{array}$ & .101 & -.133 & .022 & -.031 & 1.000 \\
\hline & & Sig. (2-tailed) & .785 & .315 & 189 & .834 & .822 & . \\
\hline
\end{tabular}

**. Correlation is significant at the 0.01 level (2-tailed).

\section{DISCUSSION}

Placement of brackets plays a vital role in initiating an orthodontic treatment for a patient who mostly suffers from problems ranging from facial esthetics to mastication. 
Debonding is a commonly encountered phenomenon that occurs when the brackets detach from the tooth surface inside the patient's mouth due to a variety of reasons which include chewing hard substances, biting on sticky foods, parafunctional habits, and improper lifestyle. This vicious cycle of debonding of the brackets can be initiated if the patient is not properly counseled as to the necessary changes in lifestyle.

In this study, out of the 100 participants, debonding was encountered on two occasions within 6 months with females being the most commonly affected gender which is similar to a study concluded by Liu Z and McGrath $\mathrm{CH} .{ }^{12}$ In both the genders, older age group individuals, less frequently, were met with bracket failure, which is similarly found in previous studies. ${ }^{13,14}$ Furthermore, young teenagers were most frequently presented with debonding of the brackets. This could be due to a non-compliant behavior to the altered lifestyle recommended by the orthodontist to prevent breakage of the brackets. Adults, on the other hand, are more compliant with given instructions.

The failure rate in our study is significantly higher than previous studies in the literature. ${ }^{15,16}$ One of the reasons which led to this high failure rate of the brackets includes improper technique used by the dentist to place the brackets and on the patient's end, incompliance with lifestyle modification and maintenance of oral hygiene.

Debonding of the brackets in this study was more commonly found in mandibular teeth. These results correspond to the previous studies carried out by Suhkia et $\mathrm{al}^{9}$ and Pseiner et al. ${ }^{17}$ Moreover, some studies do report that the debonding of the brackets is present equally in both the respective dentitions. ${ }^{18}$ Individuals who were internally motivated to initiate their orthodontic treatment were better able to protect their brackets from failing as compared to the externally motivated counterparts.

In our study, we concluded that premolars, more specifically, the second premolars were the teeth that were the most common culprits on which debonding occurred, both the first and the second times. Similar findings were presented by Zivko-Babic et al in which the second premolar region was the most commonly affected area where the debonding occurred. ${ }^{19}$ One of the reasons for this debonding to occur in the second premolar region could be the inadequate moisture control, which is one of the prime factors to control while bonding the teeth.

The teeth on which the debonding of the brackets least likely took place were the first molars. This contrasts with a study by Bjorn U. Zachrisson who found out that the bracket became loose and debonded most frequently in first molars along with the canines. ${ }^{20}$ Greater failure rates of debonding occurring in first molars could be due to masticatory forces exerted on it as these teeth are primarily responsible for chewing of food.

In this study, we evaluated debonding of the brackets which occurred initially and then again, the second time, along with the duration between the debonding episodes. Although the topic of this study was strong, we were still met with some limitations. Firstly, the sample size for the study was limited. Lastly, other orthodontic variables of the patients including overjet, deep bite, cross bite, and malocclusion classification could help to better predict the reasons for the debonding of the brackets.

\section{CONCLUSION}

Failure of the bracket to bond with the tooth surface mainly during an orthodontic treatment is a frequent finding that is both problematic for the patient as well as the orthodontist. It was concluded that females as compared to males were more likely to be affected by the bracket debonding during their active orthodontic treatment in the mandibular premolar region as compared to the maxillary region. Patient compliance, lifestyle modification and proper technique of placement of brackets are some of the factors to be kept in mind to increase the longevity of the brackets.

\section{CONFLICT OF INTEREST}

None to declare.

\section{FUNDING}

None

\section{ACKNOWLEDGEMENTS}

The authors would like to thank all the participants who took part in this study.

\section{AVAILABILITY OF DATA}

The data that supports the findings of this study is available from the corresponding author, upon reasonable request.

\section{AUTHORS CONTRIBUTIONS}

HS Supervisor, performed the critical review of the manuscript. SM data collection, designed the study, drafted the manuscript and performed literature search. FA data collection and drafted the manuscript. MD data collection 
and drafted the manuscript. MK drafted the manuscript and performed literature search. AL performed data analysis, drafted the manuscript and final review.

\section{REFERENCES}

1. Seehra J, Fleming PS, DiBiase AT. Orthodontics and the ideal occlusion - a review. Orthod Updat. 2009;2:45-9.

https://doi.org/10.12968/ortu.2009.2.2.45

2. Yao J, Li D-D, Yang Y-Q, McGrath CPJ, Mattheos N. What are patients' expectations of orthodontic treatment: a systematic review. BMC Oral Health. 2016;16:19. Available from: http://www.biomedcentral.com/1472-6831/16/19

https://doi.org/10.1186/s12903-016-0182-3

3. Taghavi Bayat J, Huggare J, Mohlin B, Akrami N. Determinants of orthodontic treatment need and demand: a cross-sectional path model study. Eur J Orthod. 2017;39:85-91. Available from: https://academic.oup.com/ejo/article-lookup/doi/10.1093/ejo/cjw020 https://doi.org/10.1093/ejo/cjw020

4. Hasan A. Frequency of Bracket Bond Failure in Orthodontic Patients with Normal Over Bite And Deep Bite. J Bahria Uni Med Dent Coll. 2017;7:82-5.

5. Theodorou CI, Kuijpers-Jagtman AM, Bronkhorst EM, Wagener FADTG. Optimal force magnitude for bodily orthodontic tooth movement with fixed appliances: A systematic review. American J Orthodontics and Dentofacial Orthopedics. 2019.

https://doi.org/10.1016/j.ajodo.2019.05.011

6. Barbosa IV, Ladewig V de M, Almeida-Pedrin RR, Cardoso MA, Santiago Junior JF, Conti AC de CF. The association between patient's compliance and age with the bonding failure of orthodontic brackets: a cross-sectional study. Prog Orthod. 2018;

https://doi.org/10.1186/s40510-018-0209-1

7. Brown $\mathrm{K}$. The impact of bonding material on bracket failure rate. Vital [Internet]. 2009;6:28-30.

https://doi.org/10.1038/vital1039

8. Murfitt P, Quick A, Swain M, Herbison G. A randomised clinical trial to investigate bond failure rates using a self-etching primer. Eur J Orthod. 2006;28:444-49.

https://doi.org/10.1093/ejo/cjl007

9. H R Sukhia, Rashna H Sukhia AM. Bracket de-bonding \& breakage prevalence in orthodontic patients. Pakistan Oral Dent J. 2011;31.71-5

10. Rasool G, Raza HA, Afzal F, Ijaz W SS. Frequency of bracket breakage $\&$ bond failure in patients, undergoing fixed orthodontic treatment at Khyber College of Dentistry, Peshawar. Pak Oral Dent J. 2013;33:299-302.

11. Pont HB, Özcan M, Bagis B, Ren Y. Loss of surface enamel after bracket debonding: An in-vivo and ex-vivo evaluation. Am J Orthod Dentofac Orthop. 2010;138:387.e1-387.e9. Available from:
https://linkinghub.elsevier.com/retrieve/pii/S0889540610004981 https://doi.org/10.1016/j.ajodo.2010.01.028

12. Liu Z, McGrath C, Hägg U. Changes in oral health-related quality of life during fixed orthodontic appliance therapy: An 18-month prospective longitudinal study. Am J Orthod Dentofac Orthop. 2011;139:214-9.Available from: https://linkinghub.elsevier.com/ retrieve/pii/S0889540610009388

https://doi.org/10.1016/j.ajodo.2009.08.029

13. Barbosa IV, Ladewig V de M, Almeida-Pedrin RR, Cardoso MA, Santiago Junior JF, Conti AC de CF. The association between patient's compliance and age with the bonding failure of orthodontic brackets: a cross-sectional study. Prog Orthod. 2018;19:11. https://doi.org/10.1186/s40510-018-0209-1

14. Stasinopoulos D, Papageorgiou SN, Kirsch F, Daratsianos N, Jäger A, Bourauel C. Failure patterns of different bracket systems and their influence on treatment duration: A retrospective cohort study. Angle Orthod. 2018;88:338-47. Available from: https://meridian.allenpress.com/angle-orthodontist/article/ 88/3/338/57825/Failure-patterns-of-different-bracket-systems-and https://doi.org/10.2319/081817-559.1

15. Almosa $\mathrm{N}$, Zafar $\mathrm{H}$. Incidence of orthodontic brackets detachment during orthodontic treatment: A systematic review. Pakistan J Med Sci. 2018;34:744-50.

https://doi.org/10.12669/pjms.343.15012

16. Rai AK. Evaluation of bracket failure rate in orthodontic patients bonded with and without primer. Saudi J Dent Res. 2015;6:48-53. https://doi.org/10.1016/j.sjdr.2014.08.001

17. Pseiner BC, Freudenthaler J, Jonke E, Bantleon H-P. Shear bond strength of fluoride-releasing orthodontic bonding and composite materials. Eur J Orthod. 2010;32:268-73.

https://doi.org/10.1093/ejo/cjp116

18. Marquezan M, Lau T, Rodrigues C, Sant'Anna E, Ruellas A, Marquezan M, et al. Shear bond strengths of orthodontic brackets with a new LED cluster curing light. J Orthod. 2010;37:37-42. https://doi.org/10.1179/14653121042858

19. Zivko-Babic J, Panduric J, Jerolimov V, Mioc M, Pizeta L, Jakovac M. Bite force in subjects with complete dentition. Coll Antropol. 2002;26:293-302.

20. Zachrisson BU. Direct bonding in orthodontic treatment and retention a post-treatment evaluation. Eur J Orthod [Internet]. 2007 Apr 1;29(Supplement 1):i128-34. Available from: https://academic.oup.com/ejo/article-lookup/doi/10.1093/ejo/cjl079 https://doi.org/10.1093/ejo/cjl079 